\title{
Halo Kinematic Streams in the Era of Gaia
}

\author{
Paola Re Fiorentin, Anna Curir, \\ Mario G. Lattanzi and Alessandro Spagna
}

INAF - Torino Astrophysical Observatory, strada Osservatorio 20, 10025 Pino T. se, Italy email: re_fiorentin@oato.inaf.it

Abstract. We report on the discovery and characterization of a possibly new signature related to the formation of the Milky Way stellar local halo.

Keywords. Galaxy: formation, halo, kinematics and dynamics

The figure presents the angular momentum distribution of 2417 FGK subdwarfs within $3 \mathrm{kpc}$ of the Sun having $[\mathrm{Fe} / \mathrm{H}]<-1.5,|\mathrm{Z}|>1.5 \mathrm{kpc}$ and selected from the SDSS-GSCII kinematic Catalog (Re Fiorentin et al., in preparation). Stars identified with symbols other than dots represent the subset of the 5 and recovered by means of cluster analysis in velocity space.

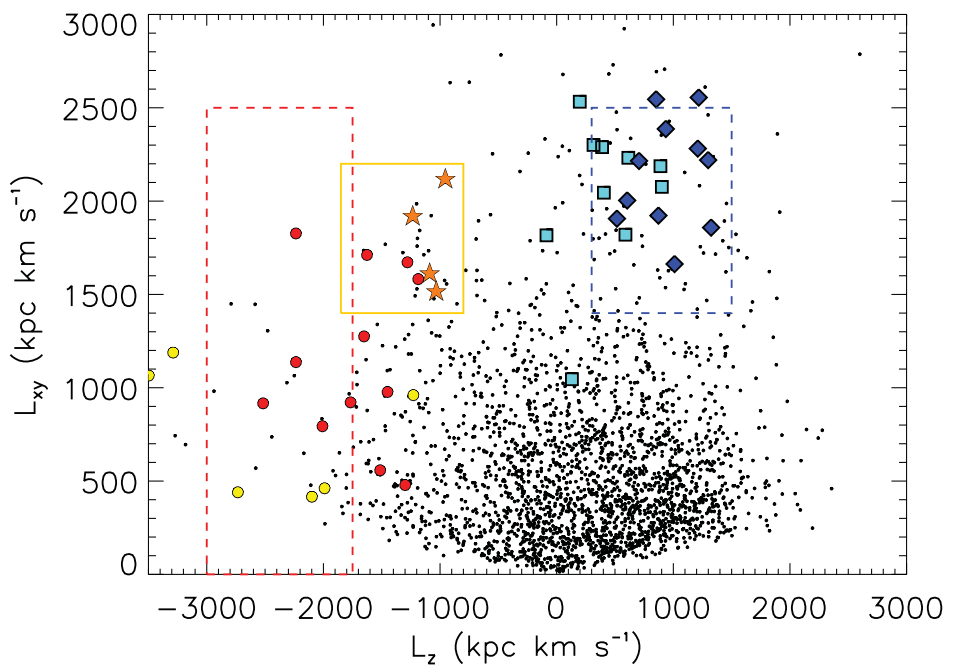

Besides new members of known streams (dashed boxes show the locus of the halo stream known to Helmi et al. 1999 and the extremely retrograde substructures found by Kepley et al. 2007), the solid box highlights a possibly new kinematic substructure: two sets of stars that are leading and trailing streams tidally torn from the body of a single dwarf galaxy on a high inclination retrograde orbit.

Gauging the impact of observational errors on our simulations (Murante et al. 2010), the prospect of highly improved data from the Gaia satellite is extremely promising and the clumpy nature of the stellar local halo will be revealed.

\section{References}

Helmi, A., White, S. D. M., de Zeeuw, P. T., \& Zhao, H. S. 1999, Nature, 402, 53

Kepley, A. A., Morrison, H. L., Helmi, A., et al. 2007, AJ, 134, 1579

Murante, G., Poglio, E., Curir, A., \& Villalobos, A. 2010, ApJ, 716, L115 